\title{
Observational consequences of optical band milliarcsecond-scale structure in active galactic nuclei discovered by Gaia
}

\author{
L. Petrov ${ }^{1,2 \star}$, and Y. Y. Kovalev ${ }^{2,3,4}$ \\ ${ }^{1}$ Astrogeo Center, 7312 Sportsman Dr., Falls Church, VA 22043, USA \\ ${ }^{2}$ Moscow Institute of Physics and Technology, Dolgoprudny, Institutsky per., 9, Moscow, Russia \\ ${ }^{3}$ Astro Space Center of Lebedev Physical Institute, Profsoyuznaya 84/32, 117997 Moscow, Russia \\ ${ }^{4}$ Max-Planck-Institut für Radioastronomie, Auf dem Hügel 69, 53121 Bonn, Germany
}

Accepted 2017 July 10. Received 2017 June 26; in original form 2017 April 21

\section{INTRODUCTION}

The European Space Agency Gaia project made a quantum leap in the area of optical astrometry. The secondary dataset of the first data release (DR1) contains positions of 1.14 billion objects (Lindegren et al. 2016) with median uncertainty 2.3 mas. Although the vast majority of Gaia detected sources are stars, over one hundred thousands of extragalactic objects, mainly active galactic nuclei (AGN), were also included in the catalogue. The only technique that can determine positions of AGNs with comparable accuracy is very long baseline interferometry (VLBI). The first insight on comparison of Gaia and VLBI position catalogues (Mignard et al. 2016; Petrov \& Kovalev 2017) revealed that the differences in VLBI/ Gaia positions are close to reported uncertainties, though a small fraction of sources $(\sim 6 \%)$ show significant offsets. We will call these sources genuine radio optical offset (GROO) objects.

We presented argumentation in Petrov \& Kovalev (2017) that unaccounted systematic errors or blunders in analysis of VLBI or Gaia data can explain offsets for some sources, but cannot explain offsets for the majority of GROO

^ E-mail: Leonid.Petrov@lpetrov.net objects. Further analysis of Kovalev et al. (2017) revealed that VLBI/Gaia offsets of a general population of radioloud AGNs, not only the matching sources with statistically significant offsets, have a preferable direction along the jet that is detected at milliarcsecond scale for the majority of radio sources (see Fig. 1). The existence of the preferable direction that is highly significant completely rules out alternative explanations of VLBI/Gaia offsets as exclusively due to unaccounted errors in VLBI or Gaia positions. Such errors, if exist, should cause either a uniform distribution of radio/optical position offsets, or have other preferable directions, for instance, across the declination axis (atmospheredriven systematic errors in VLBI) or along the predominant scanning direction (Gaia systematic errors). The preferable direction along the jet (Fig. 1) can be caused only by the intrinsic core-jet morphology. Our Monte Carlo simulation (Kovalev et al. 2017) showed that either offsets in the direction along the jet should have the mean bias exceeding 1.2 mas or the distribution of offsets should have the dispersion exceeding 2.6 mas in order to explain the histogram in Fig. 1. We should emphasize that two factors resulted in a detection of a preferable direction of VLBI/Gaia offsets: a large sample of matches and measurement of jet directions at milliarcsecond scales, which corresponds to parsec 

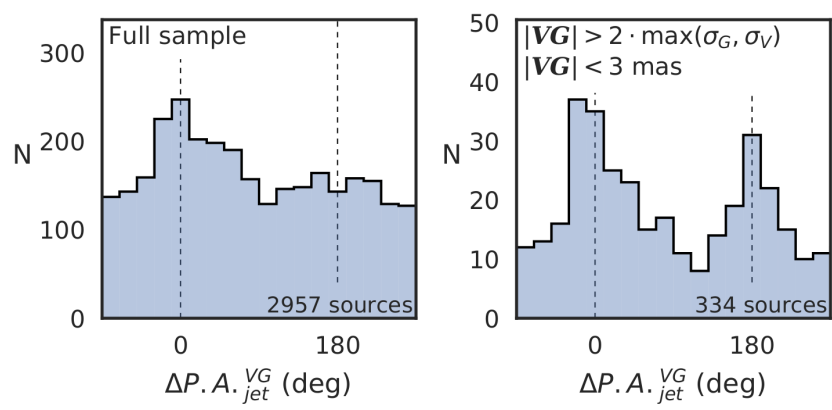

Figure 1. Histograms of direction vectors of VLBI/Gaia offsets with respect to the jet directions. The vertical dashed lines correspond to a case when the direction of the Gaia position offset with respect to the VLBI position is along the jet direction $\left(0^{\circ}\right)$ and opposite to the jet direction $\left(180^{\circ}\right)$. The left plot shows the distribution for the full sample of $2957 \mathrm{VLBI} /$ Gaia matches with the probability of false association less than $2 \cdot 10^{-4}$ and with reliably determined jet directions. The right plot shows the histogram for the sub-sample of 334 sources with offsets that a) are shorter than 3 mas, and b) longer than the maximum of both $2 \sigma$ VLBI and Gaia position uncertainties. The Figure is reproduced from Kovalev et al. (2017) with permission from Astronomy \& Astrophysics, (c) ESO.

distances. In general, jet directions at arcsecond scales (kiloparsec distances) are significantly different from directions at milliarcsecond scale (See Fig. 6 in Kharb et al. 2010). Analyzing a small sample of VLBI/Gaia matches and jet directions at arcsecond scales does not permit to reveal the systematic pattern as it was demonstrated by Makarov et al. (2017).

There are two known systematic effects that can cause a bias in VLBI positions along the jet direction and thus, contribute to the observed pattern of VLBI/Gaia position offsets at $180^{\circ}$ of the jet direction. The true jet origin, the region at the jet apex, is thought to be invisible to an observer. It is opaque and has optical depth $\tau \gg 1$ due to synchrotron self-absorption. The jet becomes visible further away from the origin when optical depth reaches $\tau \approx 1$ at the apparent jet base, we call this region the core. The higher the frequency, the closer the observed core to the jet apex (e.g., Kovalev et al. 2008; O'Sullivan \& Gabuzda 2009; Pushkarev et al. 2012; Sokolovsky et al. 2011; Kutkin et al. 2014; Kravchenko et al. 2016; Lisakov et al. 2017). This effect is called the core-shift. Kovalev et al. (2008) predicted that the apparent jet base in optical band will be shifted at 0.1 mas level with respect to the jet base at $8 \mathrm{GHz}$ opposite to the jet direction because of frequency dependence of the core-shift. However, when the core-shift depends on frequency as $f^{-1}$, it has zero contribution to the ionospherefree linear combination of group delays that is used for absolute VLBI astrometry (Porcas 2009) and thus, does not affect the absolute VLBI positions. The Blandford \& Königl (1979) model of a purely synchrotron self-absorbed conical jet in equipartition predicts the core-shift dependence on frequency $f^{-1}$. Observations (e.g. Sokolovsky et al. 2011) show no systematic deviation from this frequency dependence. The residual core-shift for the objects with the core-shift frequency dependence different than $f^{-1}$ (e.g., Kutkin et al. 2014; Lisakov et al. 2017) is over one order of magnitude too small to explain Fig. 1. In addition to the synchrotron self- absorption, an external absorption of the jet base can happen in the broad-line region or the dusty torus. It strongly depends on jet orientation (e.g., Urry \& Padovani 1995). It might further shift VLBI and/or Gaia positions along the parsec-scale jet in case if emission of the jet is significant.

The second effect is the contribution of the asymmetric radio structure to group delay that is commonly ignored in VLBI data analysis due to complexity of its computation. As we will show later, the median bias in source position caused by the neglected source structure contribution is below 0.1 mas at $8 \mathrm{GHz}$, which is also too small to explain the histograms in Fig. 1.

The remaining explanation of the observed preferential direction of VLBI/Gaia offset at $0^{\circ}$ of the jet direction is the presence of optical structure of AGNs on scales below the Gaia point-spread function (PSF) that, according to Fabricius et al. (2016), has the typical full width half maximum $($ FWHM) around $100 \times 300$ mas. Since at the moment there does not exist an instrument that could produce direct optical images at milliarcsecond resolution of objects of 15-20 magnitude, the proposed explanation can be supported only by indirect evidence.

This motivated us to consider the problem in detail and answer four questions. 1) Can the small population of known optical AGN jets at separations $0.2^{\prime \prime}-20^{\prime \prime}$ be considered as a tail of the broader population of optical jets? 2) What are the consequences of the presence of optical AGN jet structure at scales 1-200 mas that can be verified or refuted by future observations? 3) What kind of insight to AGNs physics can provide us these observational consequences? 4) How does the presence of optical structure affects the stability of AGN Gaia positions and how to mitigate them? The layout of the subsequent discussion follows this logic.

We use the following naming convention. The "core" is the apparent base of an AGN jet; its position is frequency dependent due to synchrotron self-absorption of the true base and is expected to appear further down the AGN jet with increasing observing wavelength; and the "jet" is the rest of the AGN jet structure.

\section{IMPACT OF OPTICAL JETS ON SOURCE POSITION}

As the term "active galactic nucleus" suggests, super-massive black holes (SMBHs) are assumed to be at rest in the nuclei of their host galaxies because dynamical friction against the surrounding stars and gas will eventually make an offset $\mathrm{SMBH}$ in an isolated galaxy sink to the bottom of the host galaxy gravitational potential. In the absence of strong interaction with companion galaxies, the SMBH position will coincide with the center of mass of the star population of the host galaxy. Gaia measures positions of the source's centroid. In the absence of asymmetric structures, such as optical jets, the position of the centroid in general coincides with the position of the SMBH and therefore, the Gaia position will match to the VLBI position of the core that is located in the vicinity of the SMBH. Recent galaxy mergers with SMBHs may produce massive stellar bulges containing two or more SMBHs temporarily offset in position and velocity. Extensive searches of such binary AGNs that exhibit parsec-scale radio emission revealed only two objects 
(Rodriguez et al. 2006; Condon et al. 2017) that have been firmly confirmed with VLBI observations. Thus, such objects are rare.

If the optical jet or its part is confined within the Gaia PSF, its contribution changes the position of the centroid $C_{x}$ along direction $x$ :

$$
C_{x}=\frac{\int I(x) w\left(x-x_{0}\right) x d x}{\int I(x) w\left(x-x_{0}\right) d x},
$$

where $I(x)$ is the intensity distribution along axis $x$ and $w\left(x-x_{0}\right)$ is a weighting function normalized to unity - a projection of the PSF to the direction $x$. Since the centroid depends linearly on spatial coordinates, the presence of the jet shifts the position of the centroid with respect to the core at

$$
C_{x}=\frac{\int I^{\mathrm{j}}(x) w\left(x-x_{0}\right) x d x}{\int I^{\mathrm{j}}(x) w\left(x-x_{0}\right) d x+\int I^{\mathrm{r}}(x) w\left(x-x_{0}\right) d x},
$$

where $I^{j}(x)$ is the jet intensity distribution and $I^{r}(x)$ is the remaining intensity distribution after jet subtraction. If the jet can be presented as a sum of delta-functions, and neglecting $w\left(x_{k}-x_{0}\right)-1$, which corresponds to a case when $x_{k}$ is significantly less than PSF FWHM, the expression 2 is reduced to

$$
C_{x}=\sum_{k} x_{k} \frac{F_{k}^{\mathrm{j}}}{F_{k}^{\mathrm{j}}+F_{k}^{\mathrm{r}}},
$$

where $F_{k}$ is the flux density of the $k$-th delta-function at the position $x_{k}$ and $F_{k}^{\mathrm{r}}$ is the remaining flux density excluding the $k$-th delta-function.

Fig. 2 shows schematically an AGN milliarcsecond-scale structure. The accretion disk associated with an SMBH 'A' does not necessarily coincides with the core and may be shifted with respect to the jet base. However, radio images that show the counter-jet set the limit on its displacement with respect to the jet base to a fraction of a milliarcsecond. We assume that the SMBH is located at the center of mass of a galaxy and the centroid of the hosting galaxy starlight coincides with the center of mass. This condition may not be always fulfilled in the presence of dust. The contribution of the coreshift to the VLBI position derived from dualband radio observations, the frequency-dependent vector $\overrightarrow{b v}$, is limited to the deviation of the coreshift dependence on frequency from $f^{-1}$. According to results of Sokolovsky et al. (2011), it is mostly below 0.1 mas. The contribution of source structure, being ignored, may cause a bias in the estimate of the position of the apparent jet base ' $b$ ' along the jet direction. Point ' J' in the diagram shows the centroid of an optical jet.

We do not have direct evidence that the jet base is displaced with respect to the accretion disk, but the estimates of the upper limits of such displacements mentioned above show that this is not the dominant contributor to the observed displacements. In accordance with this scheme, in general, the centroid of optical emission is determined by four parameters: flux density of the starlight $F_{\mathrm{s}}$ computed by integration of its intensity distribution; flux density of the optical core $F_{\mathrm{c}}$; flux density of the optical jet $F_{\mathrm{j}}$ produced by integration of its intensity distribution $I_{\mathrm{j}}$ and the

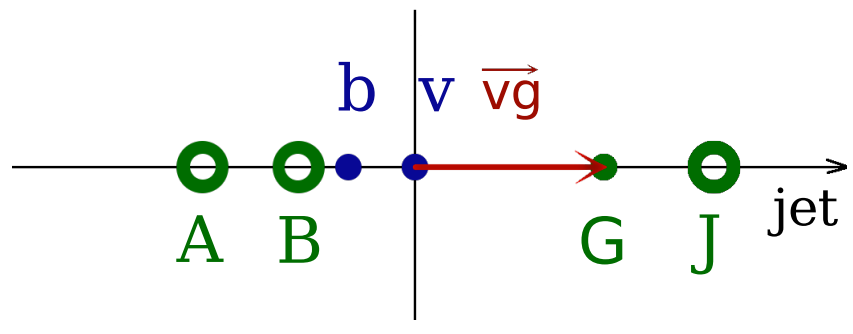

Figure 2. A simplified diagram of the AGN structure. The VLBI position is shown with ' $v$ '. It is shifted with respect to the apparent VLBI jet base $b$ (the radio core) at a given frequency due to unaccounted radio source structure contribution to its position estimate in the direction along the jet. The optical centroid 'G' is a superposition of the emission from the accretion disk 'A', apparent Gaia jet base (the optical core) 'B', and optical jet 'J'. The accretion disk is expected to be very close to the optical core. The optical jet may be absent. Astrometric observations provide us the VLBI/Gaia offset $\overrightarrow{v g}$ while VLBI imaging allows us to measure the radio parsec-scale jet direction.

displacement of its centroid with respect to the SMBH $d_{\mathrm{j}}$ (BJ vector on the diagram). Note that in a case of large offsets of optical emission centroids 'G', say greater than 1 mas, we can neglect the hypothetical displacement of the optical core 'B' with respect to the SMBH location 'A', $d_{\mathrm{c}}$. In that case, the displacement of the optical image centroid with respect to the SMBH is determined by two parameters: $r_{\mathrm{j}}=F_{\mathrm{j}} /\left(F_{\mathrm{j}}+F_{\mathrm{s}}+F_{\mathrm{c}}\right)$ and $d_{\mathrm{j}}$. According to expression 3 , $C_{x}=r_{\mathrm{j}} d_{\mathrm{j}}$. As we will show below, applying data reduction that exploits radio source images, we can determine position of point 'B' with VLBI. Then, ignoring the shift of the starlight centroid and the optical core with respect to the $\mathrm{SMBH}$, the difference VLBI/ Gaia will be equal to $C_{x}$.

\section{KNOWN LARGE OPTICAL JETS}

There are about two dozens of sources for which optical jets are detected in images with separations of $1-20^{\prime \prime}$ from galactic nuclei (f.e., Meyer et al. 2017). Since the jets are relatively weak, we can see them mainly in the sources that are at closer distances than the rest of the population. Besides, for the sources that are farther away, the angular separation of a jet from a nucleus will be smaller for a given linear separation. Jets at separations 1-20" from nuclei are not expected to affect Gaia positions since such separations are greater than the PSF. At the same time, it is instructive to get a rough estimate of how far the centroid would be shifted if sources with known optical jets were located at distances at which the jets would have been confined within the Gaia PSF. We considered three sources, 3C264, 3C273, and M87, for which we found jet photometry in the literature.

3C264 (NGC 3862, J1145+1936) is located at $z=$ 0.0216 and has a known optical jet that is extending up to $0.8^{\prime \prime}$. Using photometry of the optical jet of 3C264 presented by Lara et al. (1999), we got the estimates of the contribution of visible jet to the centroid: 15.6 mas. Independently, we used the archival Hubble Space Telescope $(H S T)$ image with the ACS/WFC instrument at $606 \mathrm{~nm}$ observed on August 21, 2015 (see Fig. 3) and computed the differences in the centroid position within the area $0.15^{\prime \prime}$ around the core 


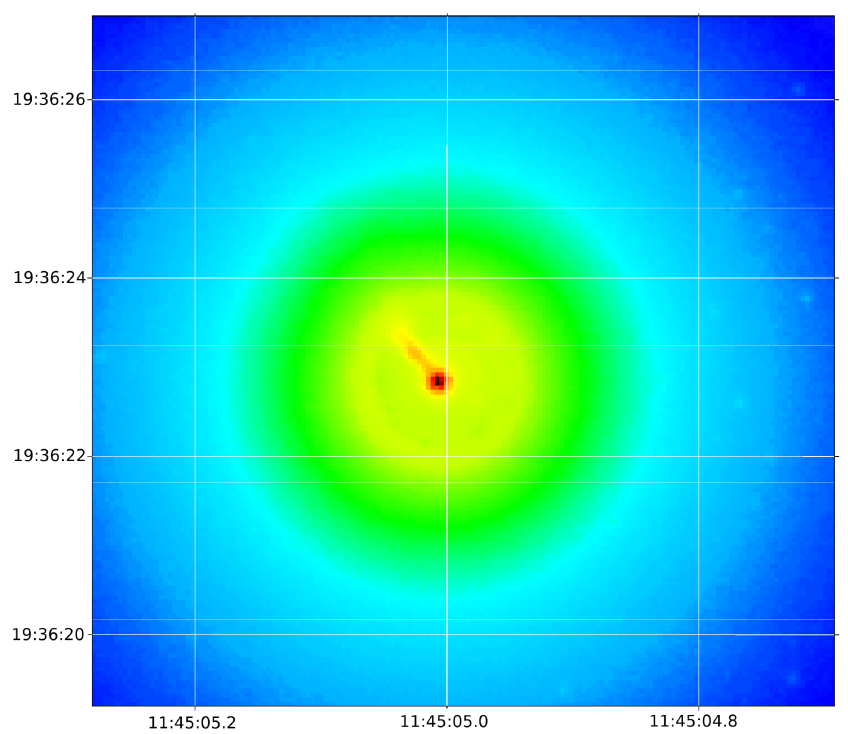

Figure 3. The archival $H S T$ image of $3 \mathrm{C} 264$ at $606 \mathrm{~nm}, H S T$ project ID 13327 (Meyer et al. 2015).

and within the whole image. The centroid difference was 14.7 mas. At $z=0.067$ this optical jet would not have been resolved by the HST, but being confined in the Gaia PSF, it would have caused a centroid shift of 5 mas.

$3 \mathrm{C} 273(\mathrm{~J} 1229+0203)$ is located at $z=0.158$ and has the optical jet that is traced to $22^{\prime \prime}$. Using the photometry of Bahcall et al. (1995), we found that the contribution of the visible part of the jet to the centroid is 19 mas.

$\mathrm{M} 87(\mathrm{~J} 1230+1223)$ at $z=0.0046$ has a rich jet structure that is traced from distance of $0.8^{\prime \prime}$ up to $26^{\prime \prime}$. Using photometry of Perez-Fournon et al. (1988) and Perlman et al. (2011), we found that the contribution of the visible part of the jet to the centroid is 56 mas. At $z=0.3$ the brightest components $\mathrm{A}, \mathrm{B}$, and $\mathrm{C}$ would be within $0.3^{\prime \prime}$ of the core and the contribution of the optical jet to the centroid position would be 1.2 mas.

Examples of 3C264 and M87 show that if these sources be farther, at a distance that direct optical observations would not have been able to resolve their jets, the shift of the centroid with respect to the core due to the presence of the jet would be several mas - close to what VLBI/Gaia comparison shows (Kovalev et al. 2017). This does not prove our interpretation of the observed preference of the VLBI/ Gaia offset directions, but it demonstrates that properties of known optical jets permit such an interpretation. We hypothesize that the known extended jets are just the tail of the distribution with the bulk of optical jets being too short and too faint to be resolved from cores even at HST images.

In these examples we counted only a visible part of the jet at distances farther than 0.15 mas. A jet or its part with the centroid at 100 mas with respect to the SMBH and with the flux density at a level of $1 \%$ of the total flux density shifts the Gaia image centroid by 1 mas. Perlman et al. (2010) present convincing argumentation that optical and radio emission is caused by the same synchrotron mechanism. Synchrotron emission in the radio range is traced from scales of ten microarcseconds to scales of arcminutes. There- fore, we conclude that the optical emission is not limited to scales of arcseconds where it could be detected with direct imaging but should be present at milliarcsecond scales as well.

\section{IMPACT OF RADIO JETS ON SOURCE POSITION}

Comparison of optical jets with radio jets at arcsecond resolutions shows that, in general, they are cospatial (e.g., Gabuzda et al. 2006). See also Kharb et al. (2010) for discussion of the misalignment between the pc-scale and kpc-scale jets in radio. The questions arises why the presence of the core does not shift VLBI and Gaia positions the same way? There are three possible reasons. First, starlight contributes in the optical range, but does not contribute significantly in the radio range. For instance, if we subtract starlight, the contribution of the optical jet and the core would have shifted the centroid of M87 by 7-9" (computed using Table 1 in Perlman et al. 2011). There is no evidence that the starlight can cause a shift of the optical centroid downstream the jet. Second, since radio spectrum of a jet and a core are different, the ratio of the flux density that comes from the radio jet to the flux density that comes from the radio core extrapolated to the optical band should be different than in the radio range. Models of synchrotron parsec-scale jet emission (e.g., Mimica et al. 2009) predict that regions downstream the apparent jet base have steep spectra. Assuming the same Doppler boosting, optical synchrotron jet emission is expected to have lower surface brightness than the radio one. Third, VLBI does not provide the position of the centroid. This requires further clarification.

The response of a radio interferometer, the complex visibility function $V_{12}$, according to the Van Zitter-Zernike theorem (Thompson et al. 2017), is

$V_{12}\left(b_{x}, b_{y}, \omega\right)=e^{i \omega \tau_{0}} \int_{-\infty}^{+\infty} I(x, y, \omega) e^{-i \omega\left(x b_{x}+y b_{y}\right)} d x d y$

where $\omega$ is the angular reference frequency of the received signal, $\tau_{0}$ - the geometric delay to the reference point on the source, and $I$ - the intensity distribution which depends on local Cartesian spatial coordinates with respect to the reference point in the image plane $x, y$, and frequency. $b_{x}$ and $b_{y}$ are the projections of the baseline vector $\vec{b}=\vec{r}_{1}-\vec{r}_{2}$ between two stations $\vec{r}_{1}, \vec{r}_{2}$ to the plane that is tangential to the center of the map $(x=0, y=0)$.

The observable used for determining source position is a group delay defined as

$$
\tau_{g r}=\frac{\partial}{\partial \omega} \arg V_{12}
$$

Typically, 10-100 estimates of group delay at different baselines at one or more epochs are used for deriving the source position. Unlike a quadratic detector installed in the focal plane of an optical telescope, e.g., a CCD camera, each given estimate of group delay of an interferometer depends on the entire image in a substantially non-linear way. A response of an interferometer, the visibility function, is proportional to a harmonic of the spatial Fourier-spectrum of the image. VLBI observations provide the spatial spectrum sampled only in 
a limited range of harmonics. For typical observations used for deriving the source positions, the range of baseline vector projections to the source's tangential plane is $80-8000 \mathrm{~km}$. This range of baseline vector projections according to the Fourier integral 4 corresponds to the range of 1-100 mas at the image plane when observations are made at $8 \mathrm{GHz}$. The interferometer is blind to spatial frequencies beyond that range due to a limited sampling of the visibility function. Features at the image smaller than that scale appear as point-like components. Features at the image larger than that scale, i.e. low surface brightness emission with variations beyond that scale, do not affect the visibilities at all.

The partial derivatives of group delay to source coordinates

$$
\frac{\partial \tau_{g r}}{\partial \alpha}=\frac{1}{c} \vec{b} \cdot \frac{\partial \vec{s}}{\partial \alpha}+O\left(c^{2}\right), \quad \frac{\partial \tau_{g r}}{\partial \delta}=\frac{1}{c} \vec{b} \cdot \frac{\partial \vec{s}}{\partial \delta}+O\left(c^{2}\right)
$$

are proportional to the baseline vector length. Here $\vec{s}$ is unit the vector of source coordinates. Therefore, despite the interferometer sees a range of spatial frequencies, the sensitivity of the interferometer to source coordinates is dominated by the longest baselines. At longest baselines, the interferometer is sensitive to the finest features of an image that is comparable to the resolution of an array. Extended features, even if they are detected by an interferometer and show up at an image, provide very small contribution to a source position estimate. Therefore, a position of an extended object derived from the analysis of interferometric observations is related not to a centroid defined by expression 1 , but to a different point.

The expression 5 can be reduced to

$$
\tau_{g r}=\tau_{o}+\tau_{\mathrm{s}},
$$

where, if we ignore dependence of source structure on frequency within the recorded band, the contribution of source structure to group delay $\tau_{\mathrm{s}}$ is expressed as

$$
\begin{aligned}
\tau_{s}\left(b_{x}, b_{y}\right) & =\frac{2 \pi}{c|\tilde{V}|^{2}} \\
& {\left[\begin{array}{l}
\operatorname{Re} \tilde{V}\left(b_{x}, b_{y}\right) \operatorname{Im}\left(\nabla \tilde{V}\left(b_{x}, b_{y}\right)\right)^{\top} \cdot\left(b_{x}, b_{y}\right)- \\
\left.\operatorname{Im} \tilde{V}\left(b_{x}, b_{y}\right) \operatorname{Re}\left(\nabla \tilde{V}\left(b_{x}, b_{y}\right)\right)^{\top} \cdot\left(b_{x}, b_{y}\right)\right] .
\end{array}\right.}
\end{aligned}
$$

Here we denote the visibility without the geometric term as $\tilde{V}$, i.e. $\tilde{V}=V_{12}\left(\tau_{0}=0\right)$.

The term $\tau_{\mathrm{s}}$ has a complicated dependence on the source image that can be expressed analytically only for some simplest cases (Charlot 1990). There are two approaches for the treatment of the $\tau_{\mathrm{s}}$ term in data analysis. The first approach is to compute $\tau_{\mathrm{s}}$ using an image. In that case the position will be related to the reference point on the image that is explicitly chosen. The second approach is to set $\tau_{\mathrm{s}}=0$ during data reduction, which is equivalent to choosing $I(x, y)=\delta(x, y)$. Term $\tau_{s}$ in general is not proportional to the partial derivatives of group delay with respect to source coordinates. Therefore, its omission is not equivalent to a shift in source positions and it will not be absorbed entirely by causing a bias in the source position estimates. Large residuals will be removed during the outlier elimination procedure; smaller residuals will propagate to the solution and affect source positions. This approach is up to now commonly adopted in all VLBI data analyses, including those used for deriving source position catalogues, since the contribution of the source structure usually does not dominate the error budget.

The magnitude of the position bias caused by ignoring $\tau_{\mathrm{s}}$ depends on many factors, including the observation schedule that affects a selection of of the Fourier transform harmonics of the source brightness distribution contributing to $\tau_{\mathrm{s}}$. For demonstrating the magnitude of the source structure contribution, we reprocessed observing session BL229AA from the VLBA MOJAVE program (Lister et al. 2016) observed on September 26, 2016. This 24-hour experiment was designed to get high fidelity images of $30 \mathrm{ob}-$ jects at $15.3 \mathrm{GHz}$. Most target sources have rich structure, i.e. the sample was biased towards the sources with significant $\tau_{\mathrm{s}}$. We performed two full data analysis runs of the BL229AA observing session: the first with $\tau_{\mathrm{s}}$ computed according to the expression 8 utilizing the images generated during processing this experiment by the MOJAVE team and made publicly available ${ }^{1}$ and the second with $\tau_{\mathrm{s}}$ set to zero. The reference point on the image was set to the image peak intensity pixel for these tests. Our analysis included fringe fitting, elimination of all outliers exceeding 3 times weighted root mean squares of residuals ( $1.2 \%$ observations) and estimation of model parameters that included station positions, the Earth orientation parameters, clock function for all stations, except the reference one, represented with Bsplines of the 1st degree, residual atmospheric path delay in zenith direction for all sites, also represented with B-spline of the 1st degree, and source coordinates. The weighted root mean squares of postfit residuals was 19.8 ps for the solution that uses $\tau_{\mathrm{s}}$ computed from the images and $21.1 \mathrm{ps}$ for the solution that set $\tau_{\mathrm{s}}$ to zero. Source position uncertainties were at a range of $40-120 \mu$ as. Table 1 shows the result sorted in increasing the contribution of source structure to source position.

Analysis of the Table 1 shows that the median position bias even for the sample of sources with rich structures is only 0.06 mas. It exceeds 0.5 mas only for two sources, J1229+0203 (3C273) and J1153+4036. Their images are shown in Fig. 4. In general, the sources with such structures are rare, less than $2 \%$. The position offset occurs predominately along the jet: either towards or opposite to the jet direction. The magnitude of the position offset has little in common with the magnitude of the shift of the centroid defined by expression 1 with respect to the brightest component of the source.

In order to illustrate further the effect of source structure on source position from VLBI observations, we ran several simulations. We used conditions and the setup of VLBA observations of $3 \mathrm{C} 273$ within the BL229AA segment of the MOJAVE program and replaced the 3C273 image with a simulated image. Then we repeated the procedure of outlier elimination and re-weighting and made two solutions with $\tau_{\mathrm{s}}$ computed from the simulated image and with $\tau_{\mathrm{s}}=0$ using exactly the same flagging and weights.

We modeled an image with two components, each with total flux density 1 Jy. We considered four cases (See Fig. 5):

(i) Both components are circular Gaussians with the

1 Available from http://www.physics.purdue.edu/MOJAVE 
Table 1. The contribution of source structure to source position estimates from processing BL229AA $15 \mathrm{GHz}$ VLBA observing session of the MOJAVE program (Lister et al. 2016). The third column shows the magnitude of the offset from the lowest to the highest values and the fourth column shows the position angle of the offset with respect to jet direction. $P A_{\mathrm{j}}=0$ corresponds to the offset towards the jet direction of the source position estimate from the solution with $\tau_{\mathrm{s}}$ applied with respect to the estimate from the solution with $\tau_{\mathrm{s}}$ set to zero. The fifth column shows the position of the image centroid with respect to the location of the image maximum.

\begin{tabular}{llcrc}
\hline J2000 & B1950 & $\begin{array}{r}|\vec{b}| \text { offset } \\
\text { name }\end{array}$ & $\begin{array}{r}P A_{\mathrm{j}} \\
(\mathrm{deg})\end{array}$ & $\begin{array}{c}\text { Centroid } \\
(\mathrm{mas})\end{array}$ \\
\hline $\mathrm{J} 0825+6157$ & $0821+621$ & 0.01 & -76 & 0.17 \\
$\mathrm{~J} 0510+1800$ & $0507+179$ & 0.01 & -98 & 0.07 \\
$\mathrm{~J} 0259+0747$ & $0256+075$ & 0.03 & -174 & 0.16 \\
$\mathrm{~J} 0309+1029$ & $0306+102$ & 0.03 & -162 & 0.10 \\
$\mathrm{~J} 2152+1734$ & $2150+173$ & 0.03 & 114 & 0.45 \\
$\mathrm{~J} 0505+0459$ & $0502+049$ & 0.04 & -157 & 0.25 \\
$\mathrm{~J} 1031+7441$ & $1027+749$ & 0.04 & 179 & 0.10 \\
$\mathrm{~J} 1603+5730$ & $1602+576$ & 0.04 & 91 & 0.33 \\
$\mathrm{~J} 1848+3244$ & $1846+326$ & 0.04 & -131 & 0.68 \\
$\mathrm{~J} 0854+2006$ & $0851+202$ & 0.04 & -76 & 0.07 \\
$\mathrm{~J} 0017+8135$ & $0014+813$ & 0.05 & 127 & 0.17 \\
$\mathrm{~J} 1551+5806$ & $1550+582$ & 0.05 & 123 & 0.13 \\
$\mathrm{~J} 0131+5545$ & $0128+554$ & 0.06 & 163 & 1.05 \\
$\mathrm{~J} 1835+3241$ & $1833+326$ & 0.06 & -102 & 0.76 \\
$\mathrm{~J} 2042+7508$ & $2043+749$ & 0.06 & -160 & 0.47 \\
$\mathrm{~J} 2301-0158$ & $2258-022$ & 0.08 & 122 & 0.12 \\
$\mathrm{~J} 0642+6758$ & $0636+680$ & 0.08 & 132 & 0.13 \\
$\mathrm{~J} 1553+1256$ & $1551+130$ & 0.08 & -9 & 1.98 \\
$\mathrm{~J} 2202+4216$ & $2200+420$ & 0.09 & 170 & 0.92 \\
$\mathrm{~J} 0925+3127$ & $0922+316$ & 0.09 & -179 & 0.91 \\
$\mathrm{~J} 0214+5144$ & $0210+515$ & 0.09 & -155 & 0.47 \\
$\mathrm{~J} 2016+1632$ & $2013+163$ & 0.10 & 105 & 0.18 \\
$\mathrm{~J} 0839+1802$ & $0836+182$ & 0.11 & 178 & 1.56 \\
$\mathrm{~J} 1925+1227$ & $1923+123$ & 0.12 & 20 & 0.06 \\
$\mathrm{~J} 1145+1936$ & $1142+198$ & 0.12 & 149 & 0.56 \\
$\mathrm{~J} 1756+1535$ & $1754+155$ & 0.14 & -13 & 0.19 \\
$\mathrm{~J} 1719+1745$ & $1717+178$ & 0.19 & -155 & 0.22 \\
$\mathrm{~J} 1421-1118$ & $1418-110$ & 0.22 & 1 & 0.01 \\
$\mathrm{~J} 1229+0203$ & $1226+023$ & 0.51 & -67 & 2.58 \\
$\mathrm{~J} 1153+4036$ & $1151+408$ & 2.40 & -157 & 1.06 \\
\hline
\end{tabular}

FWHM 0.05 mas, i.e. unresolved for BL229AA experiment. The separation of components is 10 mas.

(ii) The first component in the center of the field is a circular Gaussian with the FWHM 0.05 mas, and the second displaced component is a circular Gaussian with the FWHM 1.0 mas. The separation of components is 10 mas. For comparison, the beam has FWHM size of $0.3 \times 1.0$ mas.

(iii) The first component in the center of the field is a circular Gaussian with the FWHM 1.0 mas, and the second component is a one-sided elliptical Gaussian at the same center as the first component and the FWHM 1 mas along the declination axis and 5 mas along the right ascension axis. The one-sided Gaussian is zero for $x<0.0$, i.e. towards a decrease in right ascensions.

(iv) The first component in the center of the field is a circular Gaussian with the FWHM 1.0 mas, and the second one is a one-sided elliptical Gaussian at the same center with the FWHM 1 mas along the declination axis and 30 mas along the right ascension axis.
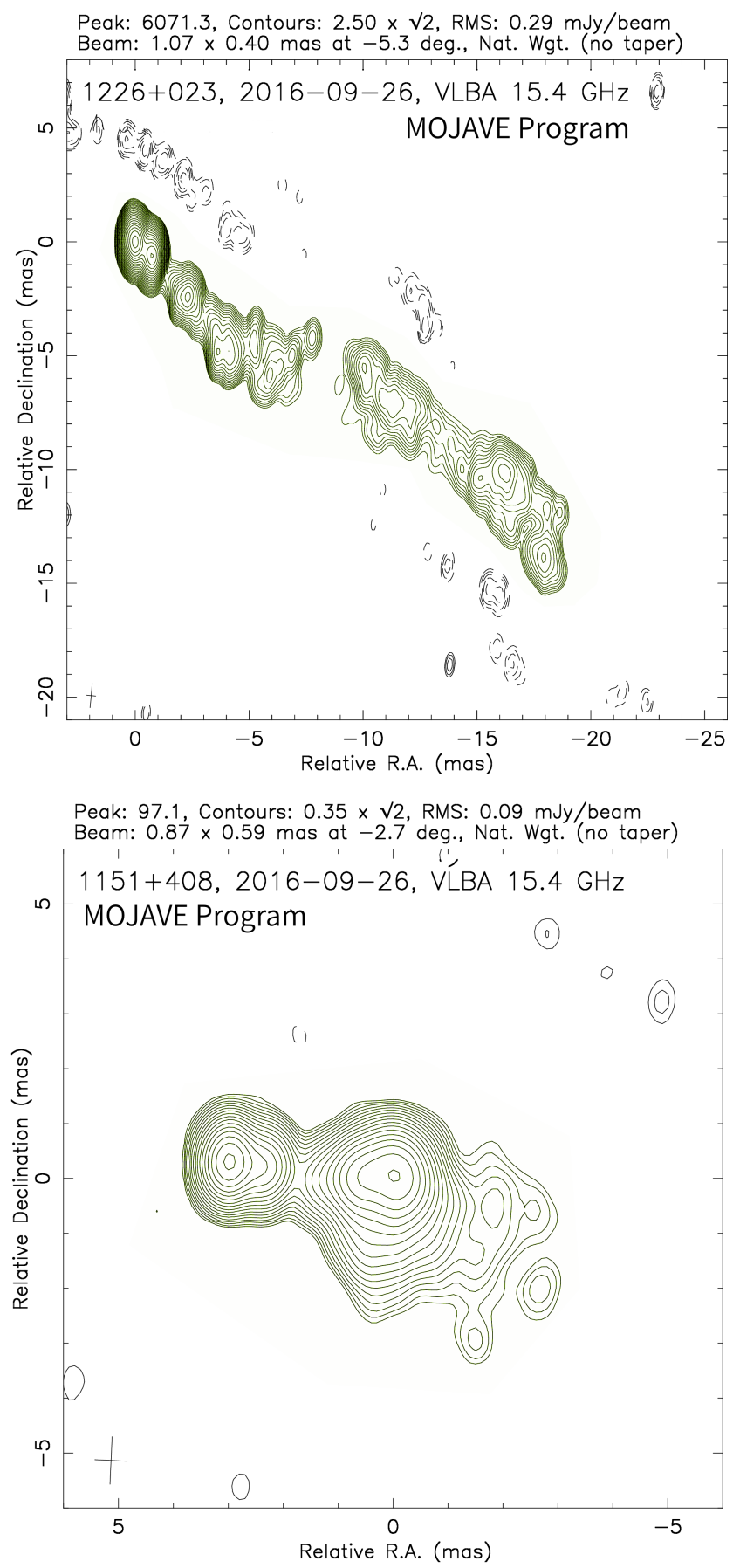

Figure 4. Images of the sources with the largest contribution of their structure to position estimates, 0.5 mas for J1229+0203 (3C273) and 2.4 mas for J1153+4036.

Table 2 shows estimates of the position offset of the solution with $\tau_{\mathrm{s}}$ computed from the modeled image with respect to the solution with $\tau_{\mathrm{s}}$ set to zero. The offset corresponds to the position bias caused by ignoring existing source structure. We see that only in a case when two components were equal unresolved Gaussians, the VLBI position estimate coincides with the centroid position. In all other cases the VLBI position estimate is very far from the centroid. The VLBI position estimate is sensitive to source structure mainly in a case when the second component has 

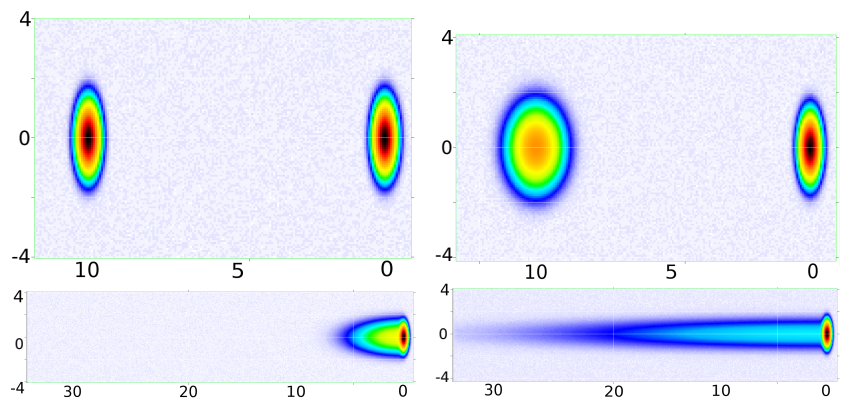

Figure 5. Simulated maps for four cases. The maps are convoluted with the beam with FWHM axes $0.3 \times 1.0$ mas. Units along the axes are milliarcseconds.

Table 2. Results of simulation. The second and third columns show position estimate differences of the solution with $\tau_{\mathrm{s}}$ computed from the simulated image with respect to the solution when $\tau_{\mathrm{S}}$ was set to zero. The fourth column shows the displacement of the image centroid with respect to the component right at the center of the simulated image.

\begin{tabular}{lrrrr}
\hline Case & \multicolumn{2}{c}{ Offset estimates } & \multicolumn{2}{c}{ Centroid offsets } \\
& $\begin{array}{r}\Delta \alpha \\
\text { mas }\end{array}$ & $\begin{array}{r}\Delta \delta \\
\text { mas }\end{array}$ & $\begin{array}{c}C_{\alpha} \\
\text { mas }\end{array}$ & $\begin{array}{c}C_{\delta} \\
\text { mas }\end{array}$ \\
\hline 1 & 5.000 & 0.0 & 5.000 & 0.000 \\
2 & 0.302 & 0.100 & 5.000 & 0.000 \\
3 & 0.153 & 0.003 & 0.857 & 0.000 \\
4 & 0.260 & 0.068 & 4.989 & 0.000 \\
\hline
\end{tabular}

size less than the interferometer resolution. It may seem counter-intuitive that the presence of source structure perfectly aligned along the right ascension axis caused position offset along declination as well. In general, $\tau_{\mathrm{s}}$ can only be partly recovered in estimates of source coordinates. The remaining source structure contribution affects the parameter estimation process like noise. It propagates to the estimates of other parameters, including declinations. We note that the contribution of actual jets to the position estimates would have been diluted even stronger since their typical shape is conical with the median apparent opening angle about $20^{\circ}$ (Pushkarev et al. 2017).

\section{KINEMATICS OF AGN JETS}

Early VLBI observations revealed that source images are changing with time (Whitney et al. 1971). Jet kinematics was extensively studied at both northern (e.g., Piner et al. 2012; Lister et al. 2016; Jorstad \& Marscher 2016) and southern hemispheres (e.g., Ojha et al. 2010). Here we provide a concise summary of the results relevant for our problem.

The intensity of the jet emission changes with time. These changes are in general frequency dependent. The intensity distribution along the jet is not uniform. The apparent jet origin (the core) is usually the brightest feature. There are areas of stronger emission or weaker emission that may not be visible on an image due to its limited dynamic range. Jets are continuous and mostly have a conical shape. Their emission steadily decreases with the distance from the core. At the same time, some jet regions (or features, compo-

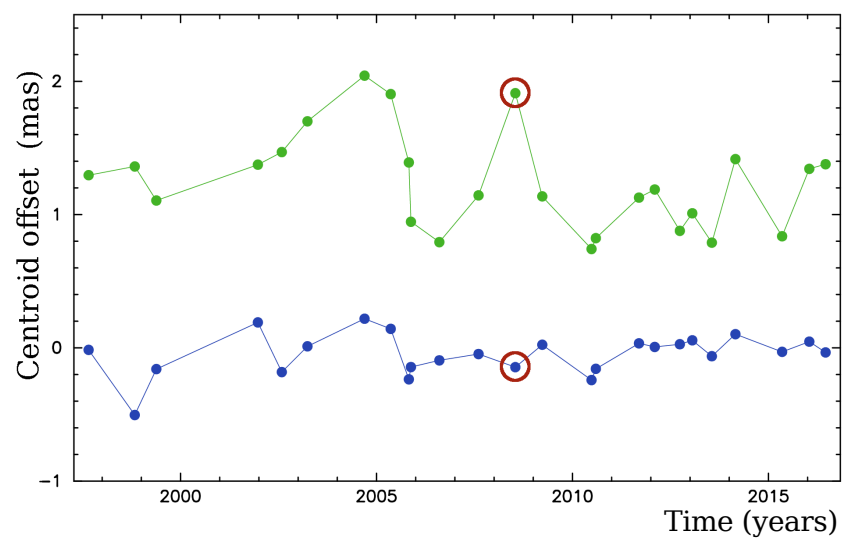

Figure 6. Evolution of the centroid offset of J1829+4844 radio images at $15.3 \mathrm{GHz}$ with respect to the core. The green points (upper part) show the centroid offsets along the jet direction. The blue points (lower part) show the centroid offsets transverse to the jet direction. The point for the epoch of image in Fig. 7 is marked with a circle.

nents, knots, blobs) might look brighter than the underlying jet. The components also dim and disappear with the distance to the core. The jet direction is stable for over decades, although ejection angle of features may vary over several tens of degrees. The typical circular standard deviation in position angle of jet components is $\sim 10^{\circ}$ (Lister et al. 2013). Jet components may appear at different parts of a jet, and typically show the radial motion (Lister et al. 2016). Some jet components are observed to have non-radial motion (Lister et al. 2016) but this does not affect the overall conical jet shapes especially for stacked multi-epoch multi-year images (Pushkarev et al. 2017). Moreover, the non-radial motion and bending accelerations tend to better align features with the inner jet (Homan et al. 2015).

According to Lister et al. (2016, Table 5), a typical angular speed of features in AGN jets at parsec scales found for the large MOJAVE sample is 0.1 mas $^{-1}$ or slower. Different components of the same jet move with approximately the same characteristic speed that represents the true flow, suggesting that the observed speed of the jet is an intrinsic property of a source being related to the underlying flow speed (Lister et al. 2013). It can rarely reach values higher than 1 mas $^{-1}$ for nearby objects. And the extreme example comes with the nearby jet in M87 which shows superluminal speed in both radio and optical band up to 25 mas $^{-1}$ (Biretta et al. 1999; Cheung et al. 2007).

Motion of bright components along the jet and changes of its flux density and the flux density of the core affect the position of the centroid. Fig. 6 demonstrates changes of the centroid offset of radio image of $\mathrm{J} 1829+4844$ at $15.3 \mathrm{GHz}$ (See its image in Fig. 7) with respect to the brightest feature that is associated with the radio core. We computed the centroid according to expression 1 using images produced by the MOJAVE team from VLBA observations. We underline that the images, not the visibility data, were used in this analysis. The changes of the centroid offset due to the source structure evolution are over 1 mas peak-to-peak along the jet direction. As expected, images at epochs with low flux density level of the core emission tend to have higher offset and, opposite to that, a flaring core decreases the offset (see the 


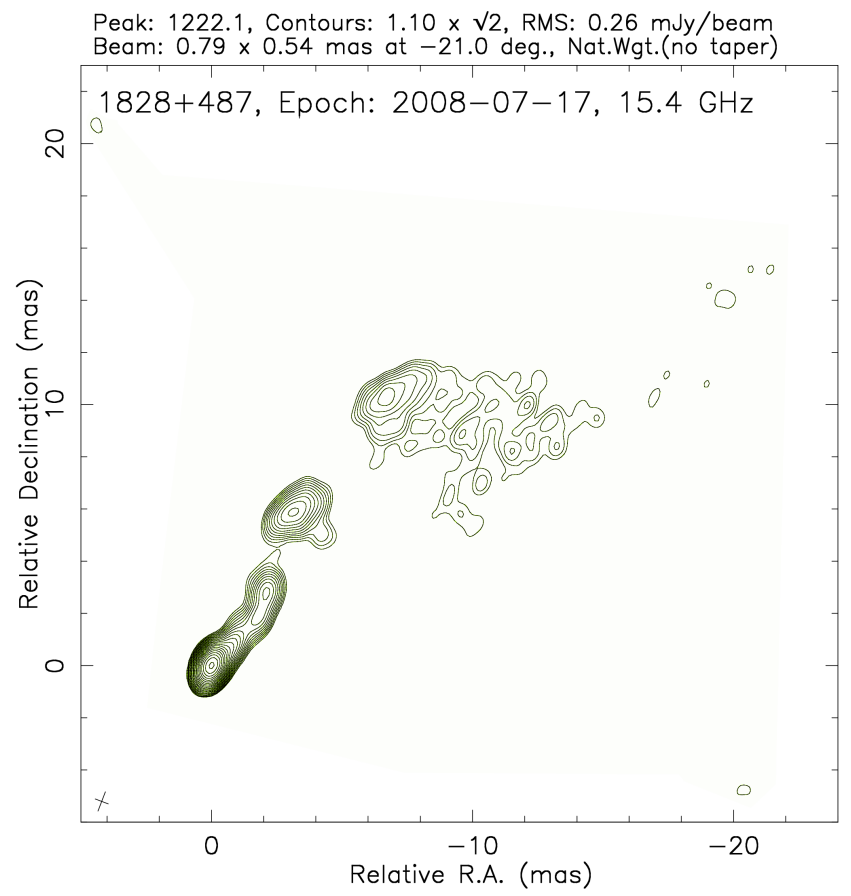

Figure 7. Image of J1829+4844 - the source with significant evolution of its radio centroid (See Fig. 6).

core modeling results in Lister et al. 2013). The root mean square (rms) of the centroid offset time series along the jet is 0.36 mas. The rms of the centroid offsets transverse the jet direction is 0.16 mas. We should note that, in general, centroid variations in optical and radio ranges are not expected to be the same since the relative weight of the core, the low surface brightness feature of jet, and the starlight are different. Fig. 6 shows what kind of changes in optical centroid may happen, provided these factors are negligible. Whether these factors are actually negligible, we do not know.

\section{EFFECT OF SOURCE FLARES}

Rapid and strong variability on time scales from decades to weeks is a distinctive intrinsic characteristics of quasars. Most AGNs with parsec-scale jets are flaring objects. An optical variability at a level of $0.3 \mathrm{mag}$ is rather common, and many sources exhibit changes exceeding one magnitude. Smith et al. (2009) provides a large numbers of light curves for many AGNs collected by the Steward Observatory spectropolarimetric monitoring project ${ }^{2}$. The position of the optical centroid is the weighted mean of the position of the starlight centroid, the accretion disk centroid, the core centroid, and the jet centroid, provided these components are within the Gaia PSF. Since during a flare the brightness of only one component increases, the ratio of fluxes of the components changes, and the centroid is shifted. It matters in what direction the optical centroid is shifted with respect to the core. Let us denote projections of the Gaia position with respect to the VLBI position on the jet direction $O_{\mathrm{j}}$ and on the direction transverse to the jet $O_{\mathrm{t}}$.

2 Project website: http://james.as.arizona.edu/ ${ }^{\text {psmith/Fermi }}$
To what extent may $O_{\mathrm{j}}$ observable change due to a flare? Let us consider a source with the jet centroid shifted with respect to the jet base at 10 mas and the flux of the jet being $20 \%$ of the total flux. According to expression 3, the source centroid is shifted at 2 mas with respect to the core. If the core flux increases by $1 \mathrm{mag}$, then $O_{\mathrm{j}}$ becomes 0.74 , i.e., decreases by 1.26 mas. If the core flux decreases by $1 \mathrm{mag}$, then $O_{\mathrm{j}}$ becomes 3.33 mas, i.e., increases by +1.33 mas. In general, changes of optical core flux by a factor of two will cause a change in the positional offset of the centroid by a factor of 1.5-3. Optical flux changes of a factor of 2, i.e., $0.75 \mathrm{mag}$, are quite common. Analysis of the correlation of radio/optical polarization (Marscher et al. 2008, 2010) suggests that most probably, these changes happen in the compact optical core at parsec scales. Therefore, we conclude that $O_{\mathrm{j}}$ changes are observable and the magnitude of the change may be close to $100 \%$ of $O_{\mathrm{j}}$ value of the quiet state.

The sign of the change is important. There are six possible cases (see Fig. 8):

1) positive projection increases by modulo $\left(O_{\mathrm{j}}+i\right)$;

2) negative projection increases by modulo $\left(O_{\mathrm{j}}-i\right)$;

3) positive projection decreases by modulo $\left(O_{\mathrm{j}}+d\right)$;

4) negative projection decreases by modulo $\left(O_{\mathrm{j}}-d\right)$;

5) positive projection is stationary $\left(O_{\mathrm{j}}+0\right)$;

6) negative projection is stationary $\left(O_{\mathrm{j}}-0\right)$.

In the first two cases we can unambiguously point in which region the flare took place: if the positive $O_{\mathrm{j}}$ increases with an increase of the total flux density, the flare occurred in the jet. If the negative $O_{\mathrm{j}}$ projection decreases even further with an increase of the total flux density, the flare occurred in the accretion disk.

The case $O_{\mathrm{j}}+d$ can be explained in two ways: a flare either in the accretion disk or in the core. The case $O_{\mathrm{j}}-d$ can also be explained in two ways: a flare either in the jet or in the core. Finally, it may happen that the centroid is stationary $\left(O_{\mathrm{j}}+0, O_{\mathrm{j}}-0\right)$. That means points A, B, J coincide and the proposed simplified scheme cannot explain the offset.

We see that analyzing correlation of the $O_{j}$ jitter and the light curve, we can get very valuable qualitative information: where the flare happened. We will show now that we are able not only to make a qualitative inference, but investigate milliarcsecond optical structure quantitatively. The dependence of the position centroid on changing brightness of the two-component model can be easily deduced from expression 3:

$$
O_{\mathrm{j}}(y)=\frac{O_{\mathrm{j}}(0)+d_{x} y}{1+y},
$$

where $y=\frac{\Delta F}{F}$ is the change of the flux density because of a flare with respect to the initial epoch $t=0$. Inverting this expression, we can find the shift of centroid of the component which flux density was constant during the flare with respect to the flaring component and its flux density $F_{f}$ :

$$
\begin{aligned}
& d_{x}(t)=F(0) \frac{O_{\mathrm{j}}(t)-O_{\mathrm{j}}(0)}{F(t)-F(0)}+O_{\mathrm{j}}(t) \\
& F_{f}(t)=O_{\mathrm{j}}(0) \frac{F(0)}{d_{x}(t)}
\end{aligned}
$$



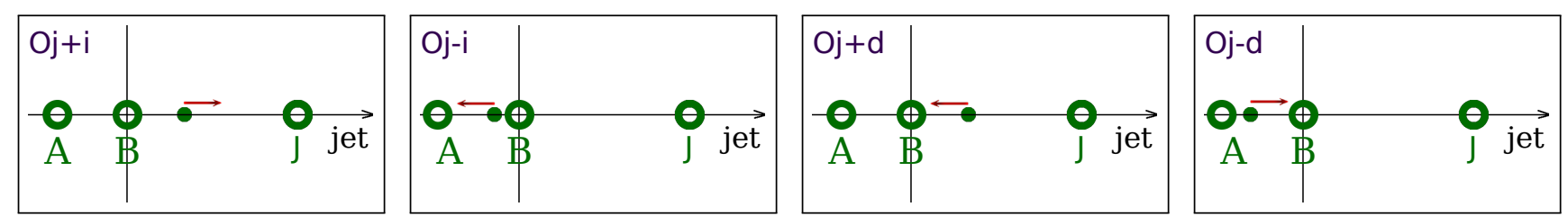

Figure 8. A simplified diagram of the $O_{\mathrm{j}}$ projection changes after a flare in the optical band: 1$) O_{\mathrm{j}}+i$ : positive projection decreases by modulo; 2) $O_{\mathrm{j}}-i$ : negative projection increases by modulo; 3) $O_{\mathrm{j}}+d$ : positive projection increases by modulo; 4 ) $O_{\mathrm{j}}-d$ : negative projection decreases by modulo. The filled circle denotes the optical centroid. The labels are the same as in Fig. 2 .

The light curves and time series of $O_{\mathrm{j}}(t)$ provide important redundant information. The stability of $d_{x}(t)$ time series will indicate that neither the flaring component, nor the component with constant flux density are moving. A statistically significant jitter of $d_{x}(t)$ will indicate that a simple stationary model does not fit the data. A straightforward interpretation of such a result as the time evolution of $d_{x}$ is problematic. If the jet centroid is moving, for instance, because of a motion of a distinctive compact feature on the jet (blob), then its flux density is changing. Analysis of radio jet kinematics shows this is a typical situation. However, jet dynamics is spawned by a process in the core. If we assume that the $i$-th jet component is moving along the jet, we have to assume that the flux density of that component, $F_{i}^{j}$ and the flux density of the core are changing. Analysis of kinematics of radio jets demonstrates that the following simplified model works most of the time (Lister et al. 2016). The core ejects components at discrete epochs. After ejection, the component moves mainly linearly. Its flux density is zero before the ejection eoch and becomes zero after some time. For such a simplified model, equations for $O_{\mathrm{j}}(t)$ and the total flux density $F_{\mathrm{t}}(t)$ are written as

$$
\begin{aligned}
O_{\mathrm{j}}(t) & =\sum_{i} \frac{v\left(t-t_{0 i}\right) F_{i}^{j}(t)+d_{i}\left(t_{0 i}\right) F_{i}^{j}\left(t_{0 i}\right)}{F_{c}(t)+\sum_{k} F_{k}^{j}(t)}, \\
F_{\mathrm{t}}(t) & =F_{c}(t)+\sum_{i} F_{i}^{j}(t), \\
F_{\mathrm{i}}^{j}(t) & =0, \quad \forall t<t_{0 i},
\end{aligned}
$$

where $F_{\mathrm{c}}(t)$ is the combined flux density of the core and starlight. $O_{\mathrm{j}}(t)$ and $F_{t}(t)$ are measurements, and $v, F_{c}(t)$, $F_{i}^{j}(t), d_{i}\left(t_{0 i}\right)$, and $t_{0 i}$ are unknowns. In general, the system does not have a unique solution, however using additional information may make this system solvable.

Let us consider a system that consists of 1) a core with variable flux density $F_{\mathrm{c}}(t)$ that includes also the contribution of starlight and 2) a jet component that moves with a constant angular velocity $v$ with variable flux density $F_{\mathrm{j}}(t)$ computed by integrating its intensity distribution. The system is observed from the moment $t_{b}$ that is not necessarily equal to the epoch of the jet component ejection $t_{0}$. For such a model the flux density of the moving jet component is expressed as

$$
\begin{aligned}
F_{\mathrm{j}}(t) & =\frac{O_{j}(t) F_{\mathrm{t}}(t)-O_{\mathrm{j}}\left(t_{b}\right) F_{\mathrm{t}}\left(t_{b}\right)}{v\left(t-t_{b}\right)}+F_{\mathrm{j}}\left(t_{b}\right) \\
F_{\mathrm{c}}(t) & =F_{\mathrm{t}}(t)-F_{\mathrm{j}}(t) \\
d_{j}(t) & =d\left(t_{b}\right)+v\left(t-t_{b}\right) .
\end{aligned}
$$

If we know the angular velocity of a component, we can determine its light curve, the light curve of the core, and the evolution of the component centroid displacement. The velocity can be derived from radio observations. This is an intrinsic property of a source that does not depend on frequency. However, expression 12 is applicable only for an interval of time when there is only one component. Determining the interval of validity of expression 12 requires utilizing additional information.

A complication arises from the fact that the Gaia position estimates of weak objects like AGNs are almost entirely derived using the data sampled along the scanning direction. A Gaia position at a given epoch is one-dimensional. Therefore, at a given time epoch the uncertainties of $O_{\mathrm{j}}$ and $O_{\mathrm{t}}$ depend on the angle between the scanning direction and the jet direction. At some epochs $O_{\mathrm{j}}$ or $O_{\mathrm{t}}$ observables may have so large uncertainties what will make them unusable for parameter estimation. Since the scanning direction changes with time due to the Gaia orbit precession, the uncertainties of the mean $O_{\mathrm{j}}$ and $O_{\mathrm{t}}$ observables mainly do not depend on scanning direction.

We should notice that the effect of source variability on position changes of objects with structure confined within the PSF is not new. It was discussed before, (f.e., Wielen 1996; Jayson 2016) in relation to the HIPPARCOS and USNO-B1.0 catalogues. As it was shown by Wielen (1996), time series of only the total flux and position displacements are sufficient for establishing the system has a structure, f.e. whether the object is binary, but are not sufficient for a separation of variables and determination of the distance between the components and their flux densities. In contrast, using $O_{\mathrm{j}}$ observables permits variable separation in a case of a simple structure, since it is based on additional information: VLBI position of the core.

\section{JITTER IN GAIA SOURCE POSITION ESTIMATES AND MITIGATION OF ITS IMPACT}

An inevitable consequence of interpretation of the observed VLBI/Gaia position differences as a manifestation of the optical jet is the non-stationarity of the centroid position determined by Gaia. Brightening of the core and, possibly, the accretion disk causes non-stationarity of the centroid. Jet kinematics, i.e., appearance and motion of new features in the jet, their motion and intensity evolution influences the position of the centroid as well. Both processes are stochastic and non-predictable. Therefore, we call it rather a jitter than a proper motion. A change in apparent position of Gaia centroids due to these processes differs from a motion of stars that is a combination of the motion in the Galactic gravity field, the orbital motion for binary or multiple system, 
and gravitational bending. Larchenkova et al. (2017) showed that micro-lensing due to randomly moving point masses in the gravitational field of the Galaxy will cause random noise in apparent position of objects located within the Galactic plane at a level of tens microarcseconds, but above that the level proper motion of stars is regular. Although proper motion of SMBH is expected to be negligible at least at the level of microarcseconds, the position of the Gaia centroid may change at the level of milliarcseconds. This change is irregular and unpredictable.

The instability of AGN position estimates derived from VLBI observations was known for a long time (f.e., Gontier et al. 2001). This instability is related to the omitted term $\tau_{\mathrm{s}}$ that accounts for source structure in data reduction. Scattering of radio emission in the interstellar medium also changes apparent radio images and may increase the errors of VLBI position estimates. This effect is most prominent in the Galactic plane (e.g., Pushkarev et al. 2013; Pushkarev \& Kovalev 2015).

The discovery of the presence of optical jets from VLBI/Gaia comparison by Kovalev et al. (2017) raises the problem of the source position jitter in the optical range. However, optical jets contribute to the centroid position differently. First, as we see from Table 1, position of the image centroid is more sensitive to the extended jet structure than the position derived from group delays. Second, the centroid position is sensitive not only to the motion of a jet component or its brightening, but more importantly to well known strong variability of the optical emission of the core or even the accretion disk without changes in the jet.

Absolute astrometry catalogues based on star observations are marred by errors that originate from uncertainties of star proper motions, which sets the limit of a catalogue accuracy (e.g., Walter \& Sovers 2000). The position accuracy degrades with time since the contribution of uncertainties in proper motions to source positions at a current epoch accumulates with time. Remote galaxies that are located so far what makes their transverse motion negligible were considered for a long time as ideal targets that are supposed to eliminate this problem (Wright 1950). The reality turned out different. Analysis of VLBI results showed that the problem of degrading position accuracy with time has gone, but a new problem appeared: position jitter due to extended parsecscale variable structure that affects position estimates. We predict a similar situation in the optical range, even at a larger scale.

The problem of the source position jitter in VLBI results can be alleviated by changing scheduling and analysis strategy. If observations are scheduled and calibrated in such a way that they can be used for generating source images, then $\tau_{\mathrm{s}}$ term can be computed and applied in data analysis. Charlot (2002) has demonstrated reduction of the source position scatter using this approach to a limited data set. Applying source structure for processing the observations collected under absolute astrometry and geodesy VLBI programs has not yet become common because it requires significant efforts and promises a little return: improvement in the source position stability at a level of a tenth of a milliarcsecond has a negligible effect on estimates of Earth orientation parameters or station positions (Xu et al. 2016) with respect to other systematic errors and it is small with respect to typical thermal noise in source positions (0.5 mas among VLBI/ Gaia counterparts, Petrov \& Kovalev 2017).

In a similar way, the problem of a source jitter in the optical centroid positions can be alleviated. First, we expect position variations to be not totally random. The position jitter will have a preferable direction along the jet, as it was established from analysis of VLBI/ Gaia position offsets (Kovalev et al. 2017). Analysis of radio jet kinematics shows that transverse jet motions are rare (Lister et al. 2016). While we expect some jitter in source positions along the jet, we expect the jitter in the transverse direction to be significantly less and probably not detectable with Gaia. Second, we expect the correlation between the centroid position jitter and the flux changes in the optical range. The larger the flux density variations, the larger the expected centroid position jitter.

Jet directions can be determined from radio observations of radio-loud AGNs. For AGNs which lack information on their jet direction from VLBI images the jet direction can be determined from analysis of their Gaia centroid time series. The scatter of the source positions in a plane tangential to the source direction can be described by a sum of two distributions: the 2D Gaussian distribution associated with errors in position time series and the distribution of the source position wander along a certain direction due to the presence of the optical jet. Fitting a straight line into the two-dimensional scatter of source position estimates with respect to the weighted mean will allow us to restore the jet direction. Since the error ellipse of Gaia positions at each individual epochs is strongly elongated across the scanning direction, the distribution of scanning directions determines whether the jet direction can be determined. If the distribution of scanning directions is substantially non-uniform, a reliable determination of jet direction even in the presence of jitter is problematic.

Analysis of $O_{\mathrm{j}}$ observables time series and optical fluxes may in some favourable cases allow us to determine the position of the optical core. If the optical jet of a two-component core-jet model is stable, which can be deduced from stability of $d_{x}(y)$ time series in expression 10 , then using the mean value of $d_{x}(y)$ and jet direction from VLBI, we will get a precise position of the optical core, which is different than the mean position of the centroid. If $d_{x}(y)$ time series show no systematic changes, determination of the optical core is possible. Since the denominator in expression 10 has the variation of the optical flux with respect to the flux at the initial epoch, the accuracy of the optical core determinations is higher when the optical flux variations are higher. Thus, the synergism of VLBI and Gaia allows us in these cases to alleviate the contribution of the jitter of the centroid position, solve for the VLBI/ Gaia bias, and determine position of the optical core. If the number of sources for which the position of the core can be determined will be high enough, these sources can be used for improvement in determination of the orientation and drift of the Gaia catalogue.

Assuming AGN position estimates are stable in time, the orientation and drift of the Gaia catalogue can be characterized by three parameters. Rotation angles, can be computed assuming the net rotation in VLBI and Gaia positions among matching sources is zero (See eq. 5 in Lindegren et al. 2016).

A small rotation that can be represented as vector $\vec{\Psi}$ 
with Cartesian coordinates $\Psi_{1}, \Psi_{2}, \Psi_{3}$ applied to an objects with polar coordinates $\alpha, \delta$ will cause increments in coordinates $\Delta \alpha, \Delta \delta$ :

$$
\begin{array}{rrrrr}
\Delta \alpha & = & -\cos \alpha \tan \delta \Psi_{1} & -\sin \alpha \tan \delta \Psi_{2} & +\Psi_{3} \\
\Delta \delta & = & \sin \alpha \Psi_{1} & -\cos \alpha \Psi_{2} &
\end{array}
$$

The coordinates of the rotation vector can be determined with least squares requiring that the position difference of matching sources with respect to VLBI be zero. In absence of the jitter, the reciprocal weights of observation equations are $1 / w_{\alpha}=\sqrt{\sigma_{\mathrm{v}}^{2}+\sigma_{\mathrm{g}}^{2}} \cos \delta$ for right ascensions and $1 / w_{\delta}=\sqrt{\sigma_{\mathrm{v}}^{2}+\sigma_{\mathrm{g}}^{2}}$ for declinations, where $\sigma_{\mathrm{v}}$ and $\sigma_{\mathrm{g}}$ are uncertainties in VLBI and Gaia positions. In order to take into account the jitter, we just inflate the position uncertainties along the jet direction:

$$
\begin{aligned}
& 1 / w_{\alpha}=\sqrt{\sigma_{\alpha, v}^{2}+\sigma_{\alpha, g}^{2}+\sigma_{j}^{2} \sin ^{2} p} \cos \delta \\
& 1 / w_{\delta}=\sqrt{\sigma_{\delta, v}^{2}+\sigma_{\delta, g}^{2}+\sigma_{j}^{2} \cos ^{2} p},
\end{aligned}
$$

where $\sigma_{\mathrm{j}}$ is the second moment of the jitter distribution along the jet and $p$ is the jet positional angle. Precise knowledge of $\sigma_{\mathrm{j}}$ is not important. Selecting $\sigma_{\mathrm{j}} \gg \max \left(\sigma_{\mathrm{v}}, \sigma_{\mathrm{g}}\right)$ will effectively down-weight the projection of the position difference along the jet, and the estimation process will use only the transverse projection in solving system 13.

\section{GALAXIES WITH WEAK JETS}

We should refrain from a generalization of results of our analysis of VLBI/Gaia offsets of the AGNs detected with VLBI to the entire population of active galaxies. The population of the AGNs selected on this basis of their parsecscale radio emission with the cutoff at $10 \mathrm{mJy}$ at $8 \mathrm{GHz}$ is biased towards relativistically-boosted jets with small viewing angles (e.g., Cohen et al. 2007; Hovatta et al. 2009; Pushkarev et al. 2017) resulting in the effects reported by Kovalev et al. (2017) and discussed in this paper. Kellermann et al. (2016) showed that for roughly $80 \%$ objects in the complete optically-selected sample of quasars their $6 \mathrm{GHz}$ radio emission from star-forming regions dominates, rather than from the synchrotron radiation of jets. Since emission from star-forming regions is much weaker, these objects are radio-quiet. Thus, the majority of the Gaia AGNs that are selected on the basis of their optical flux with the cutoff at $20.7^{m}$ are radio-quiet with radio emission from jets extremely weak or even absent. Considering argumentation of Perlman et al. (2010) that radio and optical jet emission is caused by the same mechanism, we conclude that optical jets of the radio-quiet AGNs sample are expected to be also extremely weak or even absent. At the same time, previous studies have demonstrated (see, e.g., Elvis et al. 1994; Koratkar \& Blaes 1999; Sazonov et al. 2004) that optical emission of the accretion disk and/or the host galaxy dominates for the population of AGNs selected on the basis of their optical fluxes. Consequently, the Gaia-selected AGNs should have a much smaller share of objects with significant emission of the jet than the VLBI-selected ones.

If to exclude emission from the optical jet and consider only the contribution from the accretion disk and from the starlight of the host galaxy, the optical centroid position will be affected by the displacement of the starlight centroid with respect to the accretion disk. For galaxies that do not interact with nearby companions and have no asymmetries, such as dust bars, these two points are expected to be very close, and the accretion disk variability should cause very small centroid displacements. Though, Popović et al. (2012) argue that perturbations in the inner structure of the accretion disk and surrounding dusty torus may reach a milliarcsecond level for luminous AGNs at small redshifts. To which extent these points are close, will be seen from analysis of the correlation of light curves with position time series.

In general, the positions of the radio-quiet AGNs are expected to be more stable than the positions of the radio loud sample since the contribution of one of the factors that affects position stability, the optical jet, is excluded. The position accuracy of the radio-quiet AGN sample may be the higher the position accuracy of the radio-loud AGN sample, but unfortunately, currently there is no practical way to obtain precise coordinates of such objects with VLBI and use them for radio/optical ties. In this context, the distinction between two AGNs populations is drawn based on whether the synchrotron emission dominates in the total flux density (radio-loud) or not (radio-quiet).

\section{FUTURE OBSERVATIONS}

Before the Gaia launch, it was considered for a long time that the main obstacle for VLBI/Gaia comparison would be a small number of suitable extragalactic radio sources. Dedicated programs for VLBI observations of several hundreds new suitable candidates for matching the catalogs (Bourda et al. 2011) or improving positions of several hundreds known sources (Le Bail et al. 2016) were made. It was expected that these efforts will significantly help to align the VLBI and the Gaia source position catalogues and investigate zonal errors of the catalogues.

The Gaia data release followed by the discovery of significant contribution of extended optical structure in Gaia positions (Kovalev et al. 2017) had a profound impact. First, it was found that roughly one half of the VLBI sources have a Gaia counterpart that has a weak dependence on radio flux density (Fig. 1 in Petrov \& Kovalev 2017). A dedicated search of new Gaia counterparts does not seem to be necessary. Any VLBI survey will increase the number of VLBI/Gaia matches with a rate of about one match per twothree new sources. By August 1, 2017 the total number of compact radio sources detected with VLBI under absolute astronomy programs reached 14,767. Among them, there are 7669 matches with Gaia with the probability of false association less them $2 \cdot 10^{-4}$. There will be no problem related to a shortage of matching sources for VLBI/ Gaia comparison, and the comparison itself will not be limited to an alignment of catalogues and studying zonal errors.

As we have shown, VLBI/Gaia position differences bring invaluable information. The value of this information is significantly enhanced if the jet direction is known and we can derive $O_{\mathrm{j}}$ and $O_{\mathrm{t}}$ observables. Gaia will provide time series of source positions accompanied by light curves. Analysis of $O_{\mathrm{j}}(t), O_{\mathrm{t}}(t)$ time series and light curves will be a powerful tool probing optical jets at scales two order of magnitude 
finer than the resolution of current and perspective optical telescopes. Under best conditions with no more than one evolving component, combined analysis of VLBI and Gaia will be able to provide the evolution of optical jet centroids at milliarcsecond scales.

In order to make such a deep insight into optical structure, VLBI has to solve several problems. VLBI positions of all the matches should be determined with accuracy not worse than the accuracy of Gaia. High quality radio images of matching sources should be produced. This will allow us to compute the source structure contribution and apply a correction during data reduction. Directions of jets have to be determined. We do not know in advance when a given source will have a flare. Therefore, it is desirable to have this information for all the matches (about 8,000). At the moment, the median accuracy of the VLBI position catalogue RFC_2017A $\mathrm{A}^{3}$ (Petrov \& Kovalev, in prep.) is 0.8 mas, while $22 \%$ of the sources have position errors exceeding 2 mas because of the thermal noise. Technically, using observations at VLBA or other large VLBI arrays, we can determine source positions with accuracy better than 0.2 mas if a given source is observed long enough. According to our analysis, systematic errors dominate beyond the 0.2 mas accuracy level.

In the past, there was no strong demand to have high position accuracy for all the sources with term $\tau_{\mathrm{s}}$ applied in data analysis and have their high fidelity images. At the moment, source images are available for $80 \%$ objects observed under absolute astrometry programs ${ }^{4}$. Of them, jet directions can be reliably determined for one half of the objects with an automatic procedure (Kovalev et al. 2017). Source images for 4412 objects (47\%) were derived from $60 \mathrm{~s}$ long snapshot observations made in one scan, which is not sufficient for achieving high imaging quality. Observing sources longer, in 3-6 scans, will increase the share of images where we can determine jet direction to over $90 \%$. We should stress that all these listed problems can be solved with existing facilities under dedicated program. At the same time, attempts to add some sources to regular geodetic VLBI observations (Le Bail et al. 2016; Shu et al. 2017) turned out only partly successful. Improvement of source position coordinates with a pace of $30-100$ sources per year is not sufficient to make a noticeable difference. Therefore, we envisage dedicated programs targeting all 8000 matches. The focus of these programs will be shifted from densification of the VLBI catalogue and finding suitable matches to refining source positions and images.

Such a large dataset of precise determinations of $O_{\mathrm{j}}$ and $O_{\mathrm{t}}$ observables will be useful for a number of applications. First, the time series of $O_{\mathrm{j}}(t), O_{\mathrm{t}}(t)$ accompanied with light curves and, if available, with a series of radio images, will be useful for deriving a model of optical jet evolution of objects of interest. $O_{\mathrm{t}}(t)$ observable will be useful for evaluation of random and systematic errors not related to the presence of optical structure. When the noise in the differences due to other factors affecting VLBI/Gaia positions is small with respect to $O_{\mathrm{j}}$, individual sources can be studied.

Second, the bulk data of mean values and standard deviations of these observables will be used for statistical stud-

3 Available at http://astrogeo.org/rfc

4 See http://astrogeo.org/vlbi_images ies correlating $O_{\mathrm{j}}$ and its evolution with other properties of AGNs. Statistical studies are possible even when accuracy of $O_{\mathrm{j}}$ observables is low and not sufficient for analysis of individual sources.

Third, a population of AGNs without radio counterparts can be studied. The jet direction can be found from the analysis of a scatter of position time series. The sources with significant asymmetry in their two-dimensional position scatter should be considered as candidates to AGNs. Correlation between $O_{\mathrm{j}}$ and the position jitter makes classification of a given source as an AGN almost certain.

Statistical analysis of $O_{\mathrm{j}}(t)$ and light curves has a potential to answer a number of interesting questions, such as how often, if ever, do flares occur in the accretion disk area; how often do flares occur in jet components; how long typical optical jets are; what is the role of jet kinematics in a jitter of optical centroids and what is the role of core variability.

\section{SUMMARY AND CONCLUSIONS}

Analysis of VLBI/Gaia positional offsets revealed they are not entirely random (Petrov \& Kovalev 2017). The presence of a preferable direction in the distribution of the offsets firmly associates them with an intrinsic property of AGNs: core-jet morphology (Kovalev et al. 2017). Since VLBI records voltage that is later cross-correlated and Gaia uses a quadratic detector, the CCD camera, the response of the instruments to source structure is fundamentally different. We have simulated, tested, and confirmed that VLBI is sensitive mainly to the position of the most compact detail, the AGN core. With a proper analysis procedure, the effect of source structure on position estimate can be reduced to below the 0.1 mas level. The contribution of the optical source structure on the centroid position derived from Gaia is usually greater due to a higher weight of the extended low surface brightness emission.

We predict a jitter in Gaia centroid position estimates for radio loud AGNs. It is mainly caused by variability of the optical core flux density relative to the slowly varying jet. The magnitude of the jitter depends on the magnitude of flux density variations and the extension of the jet. For highly variable sources it may reach several milliarcseconds. The presence of an unpredictable jitter in source positions is already known in VLBI astrometry results, but is new in the field of optical space astrometry. The radio-quiet AGNs may be more suitable for construction of a highly precise optical reference frame since they are expected to have more stable optical positions.

Using accurate astrometric VLBI position as a reference point of the stable radio jet base in an AGN, we can form new observables $O_{\mathrm{j}}$ and $O_{\mathrm{t}}$ - projections of the VLBI/ Gaia position difference on the parsec-scale jet direction and the direction transverse to the jet. We have shown that these observables and the optical light curves are a powerful tool for studying optical jets at the milliarcsecond scales, unreachable for any other instrument. Analysis of $O_{\mathrm{j}}(t)$ time series and optical light curves may allow recovering properties of the optical core-jet morphology: position of the jet centroid, its flux density, and in some simple cases kinematics. Analysis of these series has a potential to locate the region where 
the optical flare occurs: in the core, the accretion disc, or jet features.

A recognition of the fact that optical positions of radio loud AGNs cannot be considered as point-like unmovable sources at the Gaia level of positional accuracy leads to a paradigm shift in the field of high precision absolute astrometry.

The presence of optical structure at 1-2 mas level associated with relativistic jets revealed in the early Gaia data release for VLBI-selected AGNs sets the limit to which extent Gaia positions can be used for radio astronomical applications. At the accuracy level worse than that threshold, Gaia positions can be used for radio astronomy and vice versus. At the accuracy level better than that threshold, the positions divert since VLBI and Gaia "see" different parts of a complex radio-loud AGN with a bright relativisticallyboosted jet. That means a single technique cannot produce the reference frame that is suitable for every wavelength range even in principle. The Gaia DR1 has already surpassed that accuracy threshold. Further improvement in position accuracy of VLBI and Gaia will not results in a reconciliation of radio and optical positions but will results in improvement of accuracy of determination of these position differences. The differences are not solely due to errors in position estimates, but contain a valuable signal. Investigation of this signal will belong to the realm of astrophysics.

The applications that require positions of radio objects with accuracy better than 1-2 mas, such as space navigation, Earth orientation parameter measurement, determination of the orientation of the Earth's orbit from combined analysis of pulsar positions from VLBI and timing, cannot borrow coordinates of observed objects from Gaia, but will have to rely on their determination from VLBI in the foreseeable future.

\section{ACKNOWLEDGMENTS}

It is our pleasure to thank Claus Fabricius and Eduardo Ros for a thorough review of the manuscript and valuable suggestions that have helped to improve the manuscript. We would like to thank Sergei Sazonov and Ian Browne for fruitful discussions.

This project is supported by the Russian Science Foundation grant 16-12-10481. This work has made use of data from the European Space Agency (ESA) mission Gaia ${ }^{5}$, processed by the Gaia Data Processing and Analysis Consortium $\left(\mathrm{DPAC}^{6}\right)$. Funding for the DPAC has been provided by national institutions, in particular the institutions participating in the Gaia Multilateral Agreement. This research has made use of data from the MOJAVE database that is maintained by the MOJAVE team (Lister et al. 2009) Some of the data presented in this paper were obtained from the Mikulski Archive for Space Telescopes (MAST). STScI is operated by the Association of Universities for Research in Astronomy, Inc., under NASA contract NAS5-26555. Support for MAST for non-HST data is provided by the NASA Office of Space Science via grant NNX09AF08G and by other

5 https://www.cosmos.esa.int/gaia

6 https://www.cosmos.esa.int/web/gaia/dpac/consortium grants and contracts. We used in our work VLBA data provided by the Long Baseline Observatory that is a facility of the National Science Foundation operated under cooperative agreement by Associated Universities, Inc.

\section{REFERENCES}

Bahcall J. N., Kirhakos S., Schneider D. P., Davis R. J., Muxlow T. W. B., Garrington S. T., Conway R. G., Unwin S. C., 1995, ApJ, 452, L91

Biretta J. A., Sparks W. B., Macchetto F., 1999, ApJ, 520, 621

Blandford R. D., Königl A., 1979, ApJ, 232, 34

Bourda G., Collioud A., Charlot P., Porcas R., Garrington S., 2011, A\&A, 526, A102

Charlot P., 1990, AJ, 99, 1309

Charlot P., 2002, in Vandenberg N. R., Baver K. D., eds, International VLBI Service for Geodesy and Astrometry: General Meeting Proceedings. p. 233

Cheung C. C., Harris D. E., Stawarz Ł., 2007, ApJ, 663, L65

Cohen M. H., Lister M. L., Homan D. C., Kadler M., Kellermann K. I., Kovalev Y. Y., Vermeulen R. C., 2007, ApJ, 658, 232

Condon J. J., Darling J., Kovalev Y. Y., Petrov L., 2017, ApJ, 834, 184

Elvis M., et al., 1994, ApJS, 95, 1

Fabricius C., et al., 2016, A\&A, 595, A3

Gabuzda D. C., Rastorgueva E. A., Smith P. S., O’Sullivan S. P., 2006, MNRAS, 369, 1596

Gontier A.-M., Le Bail K., Feissel M., Eubanks T. M., 2001, A\&A, 375,661

Homan D. C., Lister M. L., Kovalev Y. Y., Pushkarev A. B., Savolainen T., Kellermann K. I., Richards J. L., Ros E., 2015, ApJ, 798, 134

Hovatta T., Valtaoja E., Tornikoski M., Lähteenmäki A., 2009, A\&A, 494, 527

Jayson J. S., 2016, MNRAS, 457, 133

Jorstad S., Marscher A., 2016, Galaxies, 4, 47

Kellermann K. I., Condon J. J., Kimball A. E., Perley R. A., Ivezić Ž., 2016, ApJ, 831, 168

Kharb P., Lister M. L., Cooper N. J., 2010, ApJ, 710, 764

Koratkar A., Blaes O., 1999, PASP, 111, 1

Kovalev Y. Y., Lobanov A. P., Pushkarev A. B., Zensus J. A., 2008, A\&A, 483, 759

Kovalev Y. Y., Petrov L., Plavin A. V., 2017, A\&A, 598, L1

Kravchenko E. V., Kovalev Y. Y., Hovatta T., Ramakrishnan V., 2016, MNRAS, 462, 2747

Kutkin A. M., et al., 2014, MNRAS, 437, 3396

Lara L., Feretti L., Giovannini G., Baum S., Cotton W. D., O’Dea C. P., Venturi T., 1999, ApJ, 513, 197

Larchenkova T. I., Lutovinov A. A., Lyskova N. S., 2017, ApJ, 835,51

Le Bail K., et al., 2016, AJ, 151, 79

Lindegren L., et al., 2016, A\&A, 595, A4

Lisakov M. M., Kovalev Y. Y., Savolainen T., Hovatta T., Kutkin A. M., 2017, MNRAS, 468, 4478

Lister M. L., et al., 2009, AJ, 137, 3718

Lister M. L., et al., 2013, AJ, 146, 120

Lister M. L., et al., 2016, AJ, 152, 12

Makarov V. V., Frouard J., Berghea C. T., Rest A., Chambers K. C., Kaiser N., Kudritzki R.-P., Magnier E. A., 2017, ApJ, 835, L30

Marscher A. P., et al., 2008, Nature, 452, 966

Marscher A. P., et al., 2010, ApJ, 710, L126

Meyer E. T., et al., 2015, Nature, 521, 495

Meyer E., et al., 2017, Galaxies, 5, 8

Mignard F., et al., 2016, A\&A, 595, A5

Mimica P., Aloy M.-A., Agudo I., Martí J. M., Gómez J. L., Miralles J. A., 2009, ApJ, 696, 1142 
O'Sullivan S. P., Gabuzda D. C., 2009, MNRAS, 400, 26

Ojha R., et al., 2010, A\&A, 519, A45

Perez-Fournon I., Gonzalez-Serrano J. I., Colina L., Biermann P. L., 1988, ApJ, 329, L81

Perlman E. S., et al., 2010, ApJ, 708, 171

Perlman E. S., et al., 2011, ApJ, 743, 119

Petrov L., Kovalev Y. Y., 2017, MNRAS, 467, L71

Piner B. G., et al., 2012, ApJ, 758, 84

Popović L. Č., Jovanović P., Stalevski M., Anton S., Andrei A. H., Kovačević J., Baes M., 2012, A\&A, 538, A107

Porcas R. W., 2009, A\&A, 505, L1

Pushkarev A. B., Kovalev Y. Y., 2015, MNRAS, 452, 4274

Pushkarev A. B., Hovatta T., Kovalev Y. Y., Lister M. L., Lobanov A. P., Savolainen T., Zensus J. A., 2012, A\&A, 545, A113

Pushkarev A. B., et al., 2013, A\&A, 555, A80

Pushkarev A. B., Kovalev Y. Y., Lister M. L., Savolainen T., 2017, MNRAS, 468, 4992

Rodriguez C., Taylor G. B., Zavala R. T., Peck A. B., Pollack L. K., Romani R. W., 2006, ApJ, 646, 49

Sazonov S. Y., Ostriker J. P., Sunyaev R. A., 2004, MNRAS, 347, 144

Shu F., et al., 2017, ApJS, 230, 13

Smith P. S., Montiel E., Rightley S., Turner J., Schmidt G. D., Jannuzi B. T., 2009, preprint, (arXiv:0912.3621)

Sokolovsky K. V., Kovalev Y. Y., Pushkarev A. B., Lobanov A. P., 2011, A\&A, 532, A38

Thompson A. R., Moran J. M., Swenson Jr. G. W., 2017, Interferometry and Synthesis in Radio Astronomy, 3rd Edition. Springer, doi:10.1007/978-3-319-44431-4

Urry C. M., Padovani P., 1995, PASP, 107, 803

Walter H., Sovers O. J., 2000, Astrometry of Fundamental Catalogues: The Evolution from Optical to Radio Reference Frames. Springer, doi:10.1007/978-3-642-57260-9

Whitney A. R., et al., 1971, Science, 173, 225

Wielen R., 1996, A\&A, 314, 679

Wright W.-H., 1950, Proc. Amer. Phil. Soc., 94, 1

Xu M. H., Heinkelmann R., Anderson J. M., Mora-Diaz J., Schuh H., Wang G. L., 2016, AJ, 152, 151

This paper has been typeset from a $\mathrm{T}_{\mathrm{E}} \mathrm{X} / \mathrm{LAT}_{\mathrm{E}} \mathrm{X}$ file prepared by the author. 